\title{
Finite-Time Consensus of Networked Multiagent Systems with Time-Varying Linear Control Protocols
}

\author{
Jiayan Wen, ${ }^{1,2}$ Chen Wang, ${ }^{2}$ Wenguang Luo, ${ }^{1}$ and Guangming Xie ${ }^{1,2}$ \\ ${ }^{1}$ College of Electrical and Information Engineering, Guangxi University of Science and Technology, Liuzhou 545006, China \\ ${ }^{2}$ Intelligent Control Laboratory, College of Engineering, Peking University, Beijing 100871, China \\ Correspondence should be addressed to Chen Wang; wangchen@pku.edu.cn
}

Received 1 February 2016; Revised 30 March 2016; Accepted 18 April 2016

Academic Editor: Peng Lin

Copyright (c) 2016 Jiayan Wen et al. This is an open access article distributed under the Creative Commons Attribution License, which permits unrestricted use, distribution, and reproduction in any medium, provided the original work is properly cited.

\begin{abstract}
Finite-time consensus problems for networked multiagent systems with first-order/second-order dynamics are investigated in this paper. The goal of this paper is to design local information based control protocols such that the systems achieve consensus at any preset time. In order to realize this objective, a class of linear feedback control protocols with time-varying gains is introduced. We prove that the multiagent systems under such kinds of time-varying control protocols can achieve consensus at the preset time if the undirected communication graph is connected. Numerical simulations are presented to illustrate the effectiveness of the obtained theoretic results.
\end{abstract}

\section{Introduction}

Multiagent systems have extensive potential applications, ranging from multiple spacecraft alignment, formation control of multiple robots, and heading direction in flocking behavior to group average in distributed computation and rendezvous of multiple vehicles. Among these, achieving consensus in networked multiagent systems has been increasingly attracting more attention in recent years, which is a comprehensive interdisciplinary research field, including control theory, mathematics, biology, physics, computer science, robot, and artificial intelligence. Great efforts have been made on the consensus problems of multiagent systems [1,2].

From the viewpoint of system and control theory, the study of consensus algorithms is mainly impelled by the particles swarm model introduced by Vicsek et al. [3]. This discrete model of finite autonomous agents assumes that all agents move in a plane with equal speed but with different headings, while each agent's heading is updated using the so-called nearest neighbor rule based on the average of its own heading plus the heading of its neighbor. Numerical simulations have been provided to demonstrate that, under their proposed rule, all agents eventually move in the same direction without the centralized coordination. Later, Jadbabaie et al. [4] gave a strict theoretical explanation of the consensus behavior of Vicsek's model and derived convergence results for several similarly inspired models. They have proven that Vicsek's model can still be valid under switching topology, but for it there does not exist a common quadratic Lyapunov function. From then on, plenty of researches have been performed on the consensus problem. Olfati-Saber and Murray [5] have introduced a systematical framework of consensus problem in networks of dynamic agents with fixed/switching topology and communication time-delays. Ren and Beard [6] have investigated a more comprehensive discrete-time consensus scheme which includes Jadbabaie's result as a special case and have presented some more relaxable conditions for consensus of information under dynamically changing interaction topologies. In [7, 8], Moreau and Lin have separately considered the more general discrete-time consensus model and continuous-time consensus model. Meanwhile, consensus problems with switching topologies and time-varying delays have been considered [9-12]. In [13-16], consensus of multiagent systems with second/higher-order dynamics has been considered. Part or all of the agents update their states according to second-order or higher-order dynamics.

In the study of consensus problem, convergence rate is an important performance index of the proposed consensus 
protocols. It has been shown in [5] that the second smallest eigenvalue of interaction graph Laplacian, called algebraic connectivity of graph, quantifies the speed of convergence of the consensus algorithm. In [17], Xiao and Boyd have considered the problem of the weight design via semidefinite convex programming so that the algebraic connectivity can be increased. Although maximizing the second smallest eigenvalue of interaction graph Laplacian allows for a better convergent rate of the linear protocols, the state consensus can never occur in finite time. In some practical situations, however, it may be required that agreement has to be reached in finite time.

The idea of finite-time convergence has been introduced to finite-time consensus for multiagent systems in [18-22]. Reference [18] has introduced the normalized and signed gradient dynamical systems associated with a differentiable function and has identified conditions that guarantee finitetime convergence. In [19], finite-time consensus tracking of multiagent systems has been reached on the terminal sliding-mode surface. Under both the global information and the local information, [20] has developed a new finitetime formation control framework for multiagent systems with a large population of members. Reference [21] has investigated finite-time consensus problems for multiagent systems and has presented a framework for constructing effective distributed protocols. In [22], weighted average consensus with respect to a monotonic function has been studied for a group of kinematic agents with time-varying topology. In the existing results, their protocols are generally discontinuous and nonlinear which, however, may not be suitable for real applications. Another shortage is the fact that only the upper bound of the convergence time is given and accurate convergence time can not be preset.

Motivated by these analyses, in this paper, we try to design a control protocol such that the consensus can be achieved at any preset time. In order to reach this goal, preset time dependent time-varying but linear feedback control protocols are presented. We find that, under the same communication conditions as those in asymptotical consensus, our control protocols work well; that is, the terminal time dependent time-varying control protocol can solve a consensus problem at any present time if the undirected communication tropology is connected.

The remaining part of this paper is organized as follows. Section 2 gives the preliminary knowledge about graph theory. Sections 3 and 4 discuss the first-order case and second-order case, respectively; both finite-time consensus control protocols are obtained. Section 5 gives the simulation results. Finally, Section 6 concludes the whole paper.

Notations. Let $\mathbb{R}$ denote the set of all real numbers. $\mathbf{1}_{N}$ represents the all 1 vector with dimension $N$. Notation $\operatorname{diag}\left\{a_{1}, \ldots, a_{N}\right\}$ represents the diagonal matrix

$$
\left[\begin{array}{ccc}
a_{1} & & 0 \\
& \ddots & \\
0 & & a_{N}
\end{array}\right] .
$$

\section{Preliminaries on Algebraic Graph Theory}

In this section, we present some definitions and properties about algebraic graph theory that will be used in this paper. For more details, we refer to $[5,23]$.

Graph will be used to describe the communication topology among agents. Let $\mathscr{G}=(\mathscr{V}, \mathscr{E}, \mathscr{A})$ be an undirected graph with the set of vertices $\mathscr{V}=\{1,2, \ldots, N\}$, the set of edges $\mathscr{E} \subseteq \mathscr{V} \times \mathscr{V}$, and a weighted adjacency matrix $\mathscr{A}=\left[a_{i j}\right]$ with nonnegative adjacency elements $a_{i j}$. An edge of $\mathscr{G}$ is denoted by $e_{i j}=(j, i)$. The adjacency elements associated with the edges are positive; that is, $e_{i j} \in \mathscr{E} \Leftrightarrow a_{i j}>0$. Moreover, we assume $a_{i i}=0$ for all $i \in \mathscr{V}$. The set of neighbors of node $i$ is denoted by $\mathcal{N}_{i}=\{j \in \mathscr{V}:(j, i) \in \mathscr{E}\}$. Since the graph is undirected, it means that once $e_{i j}$ is an edge of $\mathscr{G}, e_{j i}$ is an edge of $\mathscr{G}$ as well. As a result, the adjacency matrix $\mathscr{A}$ is a symmetric nonnegative matrix.

The degree of node $i$ is the number of its neighbors $\mathcal{N}_{i}$ and is denoted by $\operatorname{deg}(i)$. The degree of node $i$ is given by

$$
\operatorname{deg}(i)=\sum_{j=1}^{N} a_{i j}
$$

The degree matrix is defined as $\Delta=\operatorname{diag}\{\operatorname{deg}(1), \operatorname{deg}(2), \ldots$, $\operatorname{deg}(N)\}$. Then the Laplacian of graph $\mathscr{G}$ is defined by

$$
L=\Delta-\mathscr{A} \text {. }
$$

An important fact of $L$ is that all the row sums of $L$ are zero and thus $\mathbf{1}_{N}=[1,1, \ldots, 1]^{T} \in \mathbb{R}^{N}$ is an eigenvector of $L$ associated with the zero eigenvalue.

A path between distinct vertices $i$ and $j$ means a finite ordered sequence of distinct edges of $\mathscr{G}$ in the form $\left(i, k_{1}\right),\left(k_{1}, k_{2}\right), \ldots,\left(k_{l}, j\right)$. A graph is called connected if there exists a path between any two distinct vertices of the graph.

Lemma 1 (see [23]). An undirected graph $\mathscr{G}$ is connected if and only if the rank of its Laplacian matrix $L$ is $N-1$.

By Lemma 1, for a connected graph, there is only one zero eigenvalue of $L$; all the other ones are positive and real.

\section{First-Order Dynamics}

Consider a multiagent system consists of $N$ identical agents with the first-order dynamics

$$
\dot{x}_{i}(t)=u_{i}(t), \quad i=1,2, \ldots, N,
$$

where $x_{i}(t) \in \mathbb{R}$ and $u_{i}(t) \in \mathbb{R}$ are the state and the control input of the agent $i$, respectively.

We propose a time-varying linear feedback control protocol for system (4):

$$
u_{i}(t)=k(t) \sum_{j \in \mathcal{N}_{i}} \alpha_{i j}\left(x_{j}(t)-x_{i}(t)\right),
$$

where $k(t) \in \mathbb{R}$ is a time-varying feedback gain to be designed and the weights $\alpha_{i j}$, for $i, j=1,2, \ldots, N$, are assumed to be given by the interaction topology $\mathscr{G}$. 
Using the notation $x(t)=\left[x_{1}^{T}(t), \ldots, x_{N}^{T}(t)\right]^{T}$, system (4) with protocol (5) can be written into a matrix form:

$$
\dot{x}(t)=-k(t) L_{\mathscr{g}} x(t),
$$

where $L_{\mathscr{G}}$ is the Laplacian matrix of the graph $\mathscr{G}$.

For system (4), the objective of finite-time consensus is to achieve the following requirement.

Given any finite time $t_{f} \in(0,+\infty)$, system (6) satisfies that, for any initial state $x(0), x_{i}(t)-x_{j}(t) \rightarrow 0$ as $t \rightarrow t_{f}^{-}$, $\forall i, j=1,2, \ldots, N$.

If the above requirement is achieved, we say that control protocol (5) solves the finite-time consensus problem at time $t_{f}$ for system (4).

In what follows, we try to find suitable control protocol (5) to solve the finite-time consensus problem for system (6).

Consider the communication topology described by an undirected graph $\mathscr{G}$; we assume it is connected. Then there exists a nonsingular matrix $P \in \mathbb{R}^{N \times N}$ such that $P^{-1} L_{\mathscr{G}} P=$ $\operatorname{diag}\left\{0, \lambda_{2}, \ldots, \lambda_{N}\right\}$.

Let $\eta=P^{-1} x$; we have

$$
\dot{\eta}(t)=-k(t) \operatorname{diag}\left\{0, \lambda_{2}, \ldots, \lambda_{N}\right\} \eta(t) .
$$

Lemma 2. Assuming the communication topology graph $\mathscr{G}$ is undirected and connected, then control protocol (5) solves the finite-time consensus problem at time $t_{f}$ if $\eta_{i}(t) \rightarrow 0$ as $t \rightarrow t_{f}^{-}$, $i=2,3, \ldots, N$.

Proof. Without loss of generality, we assume that the first column vector of the matrix $P$ is $\mathbf{1}_{N}$. Since

$$
\lim _{t \rightarrow t_{f}^{-}} x(t)=P \lim _{t \rightarrow t_{f}^{-}} \eta(t),
$$

it follows that

$$
\lim _{t \rightarrow t_{f}^{-}} x(t)=P\left[\eta_{1}(0), 0, \ldots, 0\right]^{T}=\eta_{1}(0) \mathbf{1}_{N} .
$$

It means that

$$
x_{i}(t) \longrightarrow x^{*}=\eta_{1}(0) \quad \text { as } t \longrightarrow t_{f}^{-}, i=1,2, \ldots, N .
$$

Proposition 3. Suppose that the communication topology graph $\mathscr{G}$ is undirected and connected. Given any finite time $t_{f}$, the time-varying feedback control protocol

$$
u_{i}(t)=\frac{c}{t_{f}-t} \sum_{j \in \mathcal{N}_{i}} \alpha_{i j}\left(x_{j}(t)-x_{i}(t)\right)
$$

solves the finite-time consensus problem at time $t_{f}$ for system (4), where $c$ is a positive constant scalar.

Proof. From (7), we have

$$
\dot{\eta}_{i}(t)=\frac{-c \lambda_{i}}{t_{f}-t} \eta_{i}(t), \quad t \in\left[0, t_{f}\right) .
$$

It follows that

$$
\eta_{i}(t)=\left(\frac{t_{f}-t}{t_{f}}\right)^{c \lambda_{i}} \eta_{i}(0) .
$$

Since $c$ and $\lambda_{i}$ are positive, we have

$$
\eta_{i}(t) \longrightarrow 0, \quad \text { as } t \longrightarrow t_{f}^{-} \text {. }
$$

By Lemma 8, we know that control protocol (11) solves the finite-time consensus problem at time $t_{f}$.

Remark 4. In Proposition 3, assuming $c \lambda_{i}>1$, from (13), we have

$$
\dot{\eta}_{i}(t)=c \lambda_{i} \frac{\left(t_{f}-t\right)^{c \lambda_{i}-1}}{t_{f}^{c \lambda_{i}}} \eta_{i}(0) .
$$

This implies that $\dot{\eta}_{i}(t)$ is bounded, $\forall t \in\left[0, t_{f}\right)$. It is easy to verify that $u_{i}$ is bounded, $\forall t \in\left[0, t_{f}\right)$. This means that if we select $c$ such that

$$
c>\max _{i=2, \ldots, N} \frac{1}{\lambda_{i}}
$$

control protocol (11) is always bounded.

Proposition 5. Assuming the communication topology graph $\mathscr{G}$ is undirected and connected, given any finite time $t_{f}$, the time-varying feedback control protocol

$$
u_{i}(t)=\frac{c}{\left(t_{f}-t\right)^{m}} \sum_{j \in \mathcal{N}_{i}} \alpha_{i j}\left(x_{j}(t)-x_{i}(t)\right)
$$

solves the finite-time consensus problem at time $t_{f}$ for system (4), where $c$ is a positive constant scalar and $m \geq 1$ is a positive integer.

Proof. If $m=1$, we come back to Proposition 3. If $m>1$, we have

$$
\begin{aligned}
& \dot{\eta}_{i}(t)=-\frac{c \lambda_{i}}{\left(t_{f}-t\right)^{m}} \eta_{i}(t), \\
& \eta_{i}(t) \\
& =\exp \left(\frac{c \lambda_{i}}{(1-m)\left(t_{f}-t\right)^{m-1}}-\frac{c \lambda_{i}}{(1-m) t_{f}^{m-1}}\right) \eta_{i}(0) .
\end{aligned}
$$

It is obvious that

$$
\frac{c \lambda_{i}}{(1-m)\left(t_{f}-t\right)^{m-1}} \longrightarrow-\infty \quad \text { as } t \longrightarrow t_{f}^{-} .
$$

It means that

$$
\eta_{i}(t) \longrightarrow 0, \quad \text { as } t \longrightarrow t_{f}^{-}
$$


Remark 6. In Proposition 5, assuming $m>1$, from (19), we have

$$
\begin{aligned}
\dot{\eta}_{i}(t) & =\frac{c \lambda_{i}}{\left(t_{f}-t\right)^{m}} \\
& \cdot \exp \left(\frac{c \lambda_{i}}{(1-m)\left(t_{f}-t\right)^{m-1}}-\frac{c \lambda_{i}}{(1-m) t_{f}^{m-1}}\right) \eta_{i}(0) .
\end{aligned}
$$

Consider

$$
\begin{aligned}
& \lim _{t \rightarrow t_{f}^{-}} \frac{1}{\left(t_{f}-t\right)^{m}} \exp \left(\frac{c \lambda_{i}}{(1-m)\left(t_{f}-t\right)^{m-1}}\right) \\
& \quad=\lim _{s \rightarrow 0^{+}} \frac{1}{s^{m}} \exp \left(\frac{c \lambda_{i}}{(1-m) s^{m-1}}\right) \\
& \quad=\lim _{p \rightarrow+\infty} p^{m} \exp \left(\frac{c \lambda_{i} p^{m-1}}{(1-m)}\right)=0 .
\end{aligned}
$$

This implies that $\dot{\eta}_{i}$ is bounded, $\forall t \in\left[0, t_{f}\right)$. It follows that $u_{i}$ is bounded, $\forall t \in\left[0, t_{f}\right)$. This means that once we select $c>0$, control protocol (17) is always bounded.

In conclusion, we present the following theorem.

Theorem 7. Assuming the communication topology graph $\mathscr{G}$ is undirected and connected, given any finite time $t_{f}$, the timevarying feedback control protocol

$$
u_{i}(t)=\dot{K}(t) \sum_{j \in \mathcal{N}_{i}} \alpha_{i j}\left(x_{j}(t)-x_{i}(t)\right)
$$

solves the finite-time consensus problem at time $t_{f}$ for system (4), where function $K(t)$ satisfies that

(i) $K(t)$ is differentiable, $t \in\left[0, t_{f}\right)$;

(ii) $K(t) \rightarrow+\infty$, as $t \rightarrow t_{f}^{-}$.

Moreover, if function $K(t)$ satisfies that

(iii) $\dot{K}(t) \exp (-K(t))$ is bounded, $t \in\left[0, t_{f}\right)$, control protocol (24) is always bounded.

Proof. The conclusion is obvious since it is easy to verify that

$$
\eta_{i}(t)=\exp \left[\lambda_{i}(K(0)-K(t))\right] \eta_{i}(0) .
$$

\section{Second-Order Dynamics}

Consider a multiagent system consists of $N$ identical agents with the second-order dynamics

$$
\begin{aligned}
& \dot{x}_{i}=v_{i} \\
& \dot{v}_{i}=u_{i} \\
& \quad i=1,2, \ldots, N,
\end{aligned}
$$

where $x_{i}(t) \in \mathbb{R}, v_{i}(t) \in \mathbb{R}$, and $u_{i}(t) \in \mathbb{R}$ are the state, the velocity, and the control input of the agent $i$, respectively.

The control law studied in this section is a time-varying feedback protocol

$$
u_{i}(t)=\sum_{j \in \mathcal{N}_{i}} \alpha_{i j}\left[k_{2}(t)\left(x_{j}-x_{i}\right)+k_{1}(t)\left(v_{j}-v_{i}\right)\right],
$$

where $k_{1}(t), k_{2}(t) \in \mathbb{R}$ are time-varying feedback gains to be designed and the weights $a_{i j}$, for $i, j=1,2, \ldots, N$, are assumed to be given by the interaction topology $\mathscr{G}$.

Denote $x(t)=\left[x_{1}(t), x_{2}(t), \ldots, x_{N}(t)\right]^{T}, v(t)=\left[v_{1}(t)\right.$, $\left.v_{2}(t), \ldots, v_{N}(t)\right]^{T}$, and

$$
z(t)=x(t) \otimes\left[\begin{array}{ll}
1 & 0
\end{array}\right]^{T}+v(t) \otimes\left[\begin{array}{ll}
0 & 1
\end{array}\right]^{T} .
$$

Moreover, let

$$
\begin{aligned}
A & =\left[\begin{array}{ll}
0 & 1 \\
0 & 0
\end{array}\right], \\
B & =\left[\begin{array}{l}
0 \\
1
\end{array}\right], \\
K(t) & =\left[\begin{array}{ll}
k_{1}(t) & \left.k_{2}(t)\right] ;
\end{array}\right.
\end{aligned}
$$

system (26) with protocol (27) can be rewritten in a matrix form:

$$
\dot{z}(t)=\left[I_{N} \otimes A-L_{\mathscr{G}} \otimes B K(t)\right] z(t),
$$

where $L_{\mathscr{G}}$ is the Laplacian matrix of the graph $\mathscr{G}$.

The objective of finite-time consensus is to achieve the following requirement.

Given any finite timet $t_{f} \in(0,+\infty)$, system (30) satisfies that, for any initial state and initial speed, $x_{i}(t)-x_{j}(t) \rightarrow 0$ and $v_{i}(t)-v_{j}(t) \rightarrow 0, \forall i, j=1,2, \ldots, N$, as $t \rightarrow t_{f}^{-}$.

In what follows, we try to find suitable control protocol (27) to solve the finite-time consensus problem for system (30).

Similar to the first-order case, consider the communication topology described by an undirected graph $\mathscr{G}$, we assume it is connected, and there exists a nonsingular matrix $P \in$ $\mathbb{R}^{N \times N}$ such that $P^{-1} L_{\mathscr{G}} P=\operatorname{diag}\left\{0, \lambda_{2}, \ldots, \lambda_{N}\right\}$. Without loss of generality, we assume that the first column vector of the matrix $P$ is $\mathbf{1}_{N}$.

Let $\eta(t)=\left(P^{-1} \otimes I_{2}\right) z(t)$; then we can obtain

$$
\begin{aligned}
\dot{\eta}(t) & =\left(P^{-1} \otimes I_{2}\right) \dot{z}(t) \\
= & {\left[I_{N} \otimes A-\operatorname{diag}\left\{0, \lambda_{2}, \ldots, \lambda_{N}\right\} \otimes(B K(t))\right] \eta(t) } \\
= & \operatorname{diag}\left\{A, A-\lambda_{2} B K(t), \ldots, A-\lambda_{N} B K(t)\right\} \eta(t) .
\end{aligned}
$$

It follows that

$$
\lim _{t \rightarrow t_{f}^{-}} z(t)=\left(P \otimes I_{2}\right) \lim _{t \rightarrow t_{f}^{-}} \eta(t) .
$$

Lemma 8. Suppose that the undirected communication topology graph $\mathscr{G}$ is connected; then control protocol (27) solves the finite-time consensus problem at time $t_{f}$ if $\eta_{i}(t) \rightarrow 0$ as $t \rightarrow t_{f}^{-}$, $i=3, \ldots, 2 \mathrm{~N}$. 
Proof. From the assumption that $\eta_{i}(t) \rightarrow 0$ as $t \rightarrow t_{f}^{-}, i=$ $3, \ldots, 2 N$, we have

$$
\begin{gathered}
\lim _{t \rightarrow t_{f}^{-}} z(t)=\left(P \otimes I_{2}\right)\left[\begin{array}{c}
\eta_{1}(0)+\eta_{2}(0) t_{f} \\
\eta_{2}(0) \\
0 \\
\vdots \\
0
\end{array}\right] \\
=\mathbf{1}_{N} \otimes\left[\begin{array}{c}
\eta_{1}(0)+\eta_{2}(0) t_{f} \\
\eta_{2}(0)
\end{array}\right] .
\end{gathered}
$$

It means that

$$
\begin{aligned}
& x_{i}(t) \longrightarrow \eta_{1}(0)+\eta_{2}(0) t_{f}, \\
& v_{i}(t) \longrightarrow \eta_{1}(0),
\end{aligned}
$$

as $t \rightarrow t_{f}^{-}, i=1,2, \ldots, N$. Thus, consensus is achieved at the preset time $t_{f}$.

Lemma 9 (see [24]). Consider the linear second-order differential equation

$$
\ddot{y}(t)+p(t) \dot{y}(t)+q(t) y(t)=0, \quad t \geq 0,
$$

where $p(t)$ and $q(t)$ are given smooth functions oft. When $y_{1}(t)$ is one fundamental solution, then the other solution $y_{2}(t)$ is given by

$$
y_{2}(t)=y_{1}(t) \int_{0}^{t} \frac{W(s)}{y_{1}^{2}(s)} d s
$$

where

$$
W(t)=\exp \left(-\int_{0}^{t} p(s) d s\right)
$$

Moreover, the general solution is given by

$$
y(t)=C_{1} y_{1}(t)+C_{2} y_{2}(t)
$$

where $C_{1}$ and $C_{2}$ are constants.

Theorem 10. Assuming the undirected communication topology graph $\mathscr{G}$ is connected, for any given finite time $t_{f}$, control protocol (27) with time-varying feedback gains

$$
\begin{aligned}
& k_{1}(t)=\frac{2 c}{\left(t_{f}-t\right)^{3}}, \\
& k_{2}(t)=\frac{c}{\left(t_{f}-t\right)^{2}}
\end{aligned}
$$

solves the finite-time consensus problem at time $t_{f}$ for system (26), where $c$ is a positive constant scalar.
Proof. According to Lemma 8, we only need to show that $\eta_{i}(t) \rightarrow 0$ as $t \rightarrow t_{f}^{-}, i=3, \ldots, 2 N$. Now, let us consider the dynamics of $\eta_{i}(t), i=3, \ldots, 2 N$. It is noted that

$$
\left[\begin{array}{c}
\dot{\eta}_{2 i-1}(t) \\
\dot{\eta}_{2 i}(t)
\end{array}\right]=\left[\begin{array}{cc}
0 & 1 \\
\frac{-2 c \lambda_{i}}{\left(t_{f}-t\right)^{3}} & \frac{-c \lambda_{i}}{\left(t_{f}-t\right)^{2}}
\end{array}\right]\left[\begin{array}{c}
\eta_{2 i-1}(t) \\
\eta_{2 i}(t)
\end{array}\right] .
$$

It is easy to verify that $e^{-c \lambda_{i} /\left(t_{f}-t\right)}$ is one of the fundamental solutions for the second equation in (40). By Lemma 8, the general solution of $(40)$ is

$$
\begin{aligned}
& \eta_{2 i-1}(t)=e^{-c \lambda_{i} /\left(t_{f}-t\right)}\left\{C_{1}+C_{2} \int_{0}^{t} e^{c \lambda_{i} /\left(t_{f}-s\right)+c \lambda_{i} / t_{f}} d s\right\}, \\
& \eta_{2 i}(t)=-\frac{c \lambda_{i}}{\left(t_{f}-t\right)^{2}} \\
& \cdot e^{-c \lambda_{i} /\left(t_{f}-t\right)}\left\{C_{1}+C_{2} \int_{0}^{t} e^{c \lambda_{i} /\left(t_{f}-s\right)+c \lambda_{i} / t_{f}} d s\right\} \\
& +C_{2} e^{c \lambda_{i} / t_{f}}
\end{aligned}
$$

where $C_{1}$ and $C_{2}$ are constants.

It follows that

$$
\begin{aligned}
& \lim _{t \rightarrow t_{f}} \eta_{2 i-1}(t) \\
& =\left\{C_{1}+C_{2} \int_{0}^{t_{f}^{-}} e^{c \lambda_{i} /\left(t_{f}-s\right)+c \lambda_{i} / t_{f}} d s\right\} \lim _{t \rightarrow t_{f}} e^{-c \lambda_{i} /\left(t_{f}-t\right)}=0, \\
& \lim _{t \rightarrow t_{f}} \eta_{2 i}(t)=C_{2} e^{c \lambda_{i} / t_{f}} \\
& -\lim _{t \rightarrow t_{f}} \frac{\left(c \lambda_{i} /\left(t_{f}-t\right)^{2}\right)\left\{C_{1}+C_{2} \int_{0}^{t} e^{c \lambda_{i} /\left(t_{f}-s\right)+c \lambda_{i} / t_{f}} d s\right\}}{e^{c \lambda_{i} /\left(t_{f}-t\right)}} \\
& =C_{2} e^{c \lambda_{i} / t_{f}}-\lim _{t \rightarrow t_{f}} \frac{\left(c \lambda_{i} /\left(t_{f}-t\right)^{2}\right) C_{2} e^{c \lambda_{i} /\left(t_{f}-t\right)+c \lambda_{i} / t_{f}}}{\left(c \lambda_{i} /\left(t_{f}-t\right)^{2}\right) e^{c \lambda_{i} /\left(t_{f}-t\right)}} \\
& -\lim _{t \rightarrow t_{f}} \frac{\left(2 c \lambda_{i} /\left(t_{f}-t\right)^{3}\right)\left\{C_{1}+C_{2} \int_{0}^{t} e^{c \lambda_{i} /\left(t_{f}-s\right)+c \lambda_{i} / t_{f}} d s\right\}}{\left(c \lambda_{i} /\left(t_{f}-t\right)^{2}\right) e^{c \lambda_{i} /\left(t_{f}-t\right)}} \\
& =-\lim _{t \rightarrow t_{f}} \frac{2 c \lambda_{i}\left\{C_{1}+C_{2} \int_{0}^{t} e^{c \lambda_{i} /\left(t_{f}-s\right)+c \lambda_{i} / t_{f}} d s\right\}}{\left(t_{f}-t\right) e^{c \lambda_{i} /\left(t_{f}-t\right)}}=0 .
\end{aligned}
$$

This completes the proof.

Now, we extend the specific time-varying gain functions to a general form.

Suppose that a function $f(t)$ satisfies

(1) $f(t) \in \mathscr{C}^{2}\left[0, t_{f}\right)$, where $\mathscr{C}^{2}\left[0, t_{f}\right)$ represents a secondorder continuously differentiable function on $\left[0, t_{f}\right)$;

(2) $f(t) \rightarrow+\infty$ as $t \rightarrow t_{f}^{-}$;

(3) $\exp (-f(t))(\ddot{f}(t) / \dot{f}(t)) \rightarrow 0$ as $t \rightarrow t_{f}^{-}$. 
We consider the following time-varying gains with a general form:

$$
\begin{aligned}
& k_{1}(t)=\ddot{f}(t), \\
& k_{2}(t)=\dot{f}(t) .
\end{aligned}
$$

Then protocol (27) with (43) covers a wide range of algorithms including the specific form in Theorem 7 .

Theorem 11. Assume that the undirected communication topology graph $\mathscr{G}$ is connected. For system (26), control protocol (27) with time-varying gains (43) solves the finite-time consensus problem at any preset finite time $t_{f}$.

Proof. Similarly, we have

$$
\begin{array}{r}
{\left[\begin{array}{c}
\dot{\eta}_{2 i-1}(t) \\
\dot{\eta}_{2 i}(t)
\end{array}\right]=\left[\begin{array}{cc}
0 & 1 \\
-\lambda_{i} \ddot{f}(t) & -\lambda_{i} \dot{f}(t)
\end{array}\right]\left[\begin{array}{c}
\eta_{2 i-1}(t) \\
\eta_{2 i}(t)
\end{array}\right],} \\
i=2, \ldots, N .
\end{array}
$$

It is easy to verify that $e^{-\lambda_{i} f(t)}$ is one of the fundamental solutions for the second equation in (44). By Lemma 8, the general solution of (44) is

$$
\begin{aligned}
& \eta_{2 i-1}(t)=e^{-\lambda_{i} f(t)}\left(C_{1}+C_{2} \int_{0}^{t} e^{\lambda_{i} f(s)+\lambda_{i} f(0)} d s\right), \\
& \eta_{2 i}(t) \\
& =-\lambda_{i} \dot{f}(t) e^{-\lambda_{i} f(t)}\left(C_{1}+C_{2} \int_{0}^{t} e^{\lambda_{i} f(s)+\lambda_{i} f(0)} d s\right) \\
& \quad+C_{2} e^{\lambda_{i} f(0)},
\end{aligned}
$$

where $C_{1}$ and $C_{2}$ are constants.

It follows that

$$
\begin{aligned}
& \lim _{t \rightarrow t_{f}} \eta_{2 i-1}(t) \\
& =\lim _{t \rightarrow t_{f}} e^{-\lambda_{i} f(t)}\left(C_{1}+C_{2} \int_{0}^{t_{f}} e^{\lambda_{i} f(s)+\lambda_{i} f(0)} d s\right)=0, \\
& \lim _{t \rightarrow t_{f}} \eta_{2 i}(t)=\lim _{t \rightarrow t_{f}}-\lambda_{i} \dot{f}(t) e^{-\lambda_{i} f(t)} C_{1} \\
& +\lim _{t \rightarrow t_{f}}-\lambda_{i} \dot{f}(t) e^{-\lambda_{i} f(t)} C_{2} \int_{0}^{t} e^{\lambda_{i} f(s)+\lambda_{i} f(0)} d s \\
& +C_{2} e^{\lambda_{i} f(0)}=-C_{1} \lim _{t \rightarrow t_{f}} \frac{\lambda_{i} \dot{f}(t)}{e^{\lambda_{i} f(t)}}-C_{2} \\
& \quad \lim _{t \rightarrow t_{f}} \frac{\lambda_{i} \dot{f}(t) \int_{0}^{t} e^{\lambda_{i} f(s)+\lambda_{i} f(0)} d s}{e^{\lambda_{i} f(t)}}+C_{2} e^{\lambda_{i} f(0)}=-C_{1} \\
& \quad \lim _{t \rightarrow t_{f}} \frac{\ddot{f} f(t)}{\dot{f}(t) e^{\lambda_{i} f(t)}-C_{2}}
\end{aligned}
$$

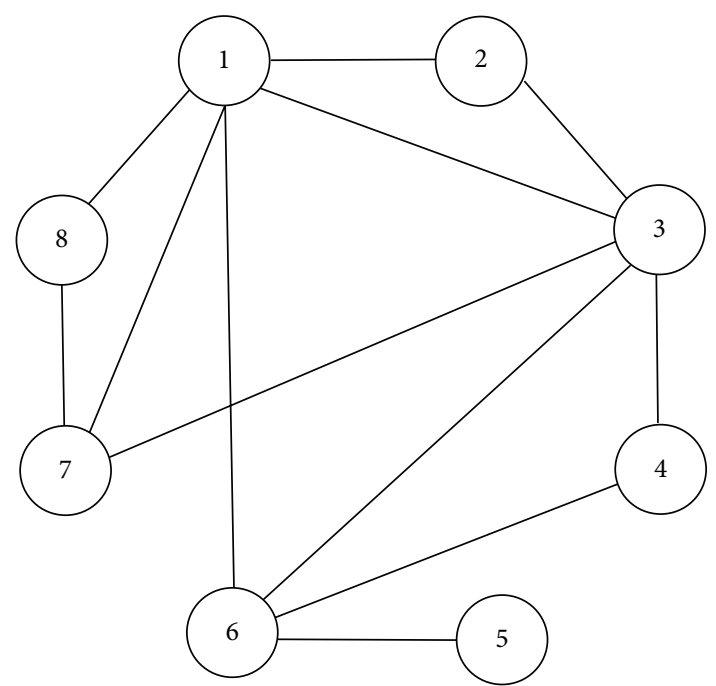

FIgURE 1: Communication graph.

$$
\begin{aligned}
& \cdot \lim _{t \rightarrow t_{f}} \frac{\ddot{f}(t) \int_{0}^{t} e^{\lambda_{i} f(s)+\lambda_{i} f(0)} d s+\dot{f}(t) e^{\lambda_{i} f(t)+\lambda_{i} f(0)}}{\dot{f}(t) e^{f(t)}} \\
& +C_{2} e^{\lambda_{i} f(0)}=-C_{2} \int_{0}^{t_{f}} e^{\lambda_{i} f(s)+\lambda_{i} f(0)} d s \\
& \cdot \lim _{t \rightarrow t_{f}} \frac{\ddot{f}(t)}{\dot{f}(t) e^{f(t)}}=0 .
\end{aligned}
$$

This completes the proof.

\section{Numerical Simulations}

To demonstrate our theoretical results in the previous two sections, we carry out numerical simulations in this section. Considering a multiagent system consisting of eight agents, the communication graph $\mathscr{G}$ is given in Figure 1.

The Laplacian matrix of $\mathscr{G}$ is given by

$$
\mathscr{L}_{\mathscr{G}}=\left[\begin{array}{cccccccc}
5 & -1 & -1 & 0 & 0 & -1 & -1 & -1 \\
-1 & 2 & -1 & 0 & 0 & 0 & 0 & 0 \\
-1 & -1 & 5 & -1 & 0 & -1 & -1 & 0 \\
0 & 0 & -1 & 2 & 0 & -1 & 0 & 0 \\
0 & 0 & 0 & 0 & 1 & -1 & 0 & 0 \\
-1 & 0 & -1 & -1 & -1 & 4 & 0 & 0 \\
-1 & 0 & -1 & 0 & 0 & 0 & 3 & -1 \\
-1 & 0 & 0 & 0 & 0 & 0 & -1 & 2
\end{array}\right] .
$$

First, we consider the dynamics of the agent with the first order. Control protocol (11) is applied as $c=2$. We run the simulations with $t_{f}=10$ and $t_{f}=1$, respectively, and show the results in Figure 2. In the simulations, the initial states of the agents are generated randomly. For ease of 


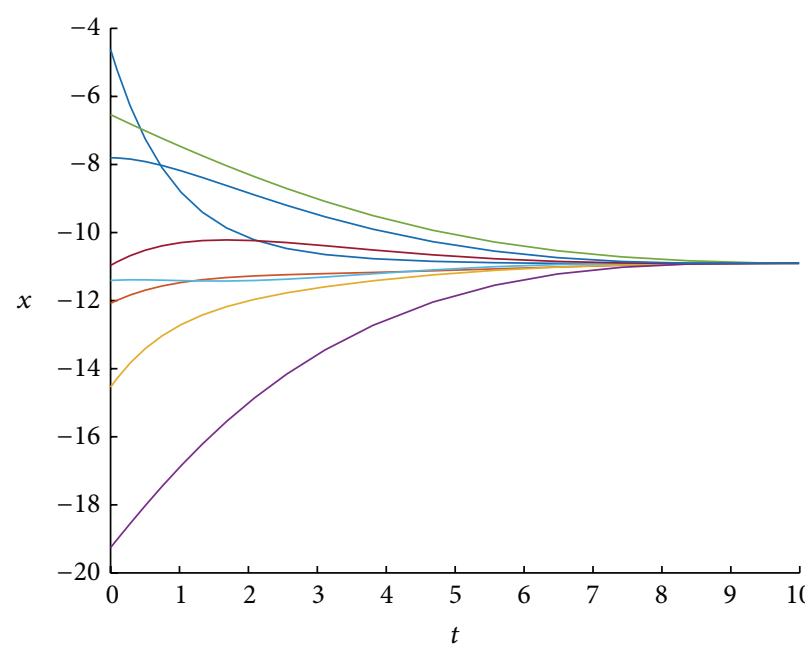

(a) $t_{f}=10$

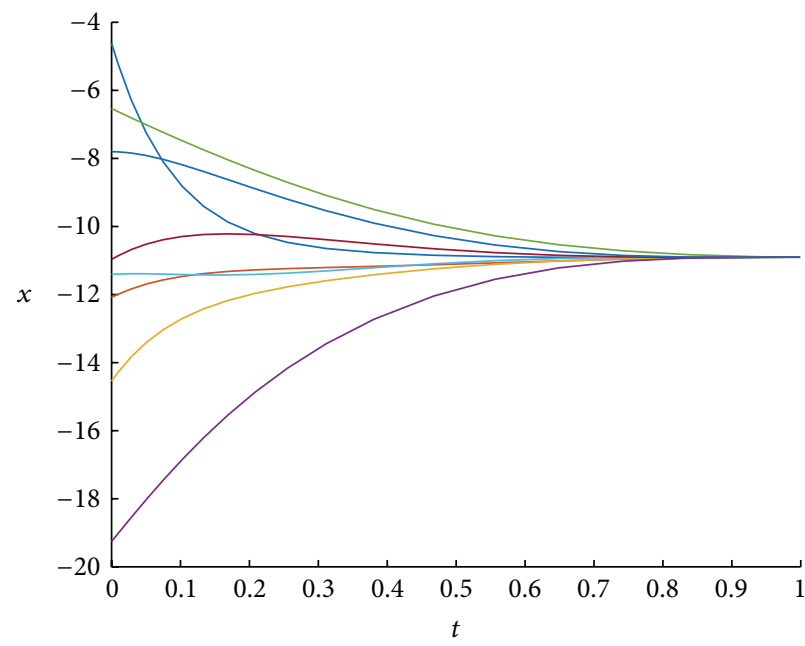

(c) $t_{f}=1$

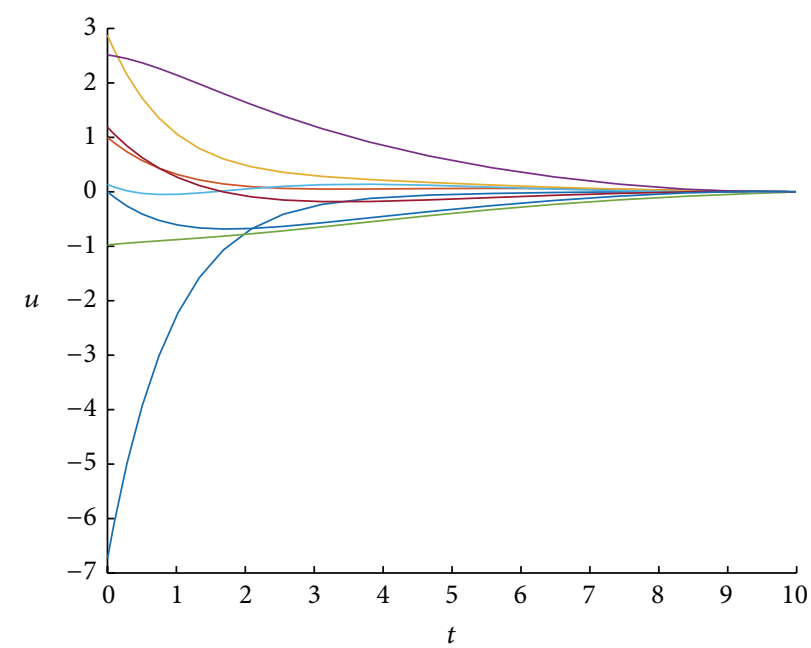

(b) $t_{f}=10$

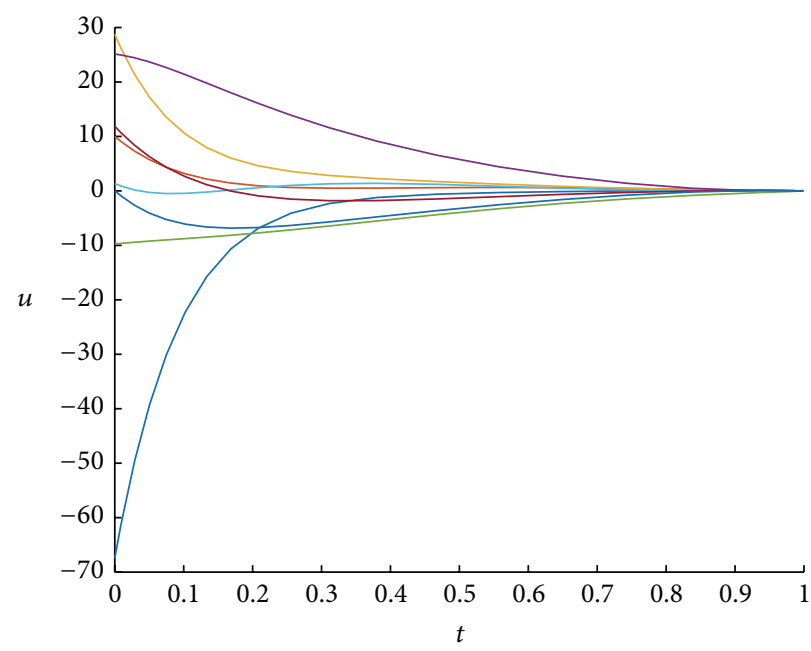

(d) $t_{f}=1$

FIGURE 2: Simulation results of control protocol (11) for the single-integrator dynamics case when $c=2$. ((a) and (b)) The case with $t_{f}=10$; ((c) and (d)) the case with $t_{f}=1 ;((a)$ and (c)) the states of all the eight agents; ((b) and (d)) the inputs of all the eight agents.

comparison, we use the same initial states of the eight agents in both simulations. The simulation results have shown that the groups of agents can reach consensus as $t \rightarrow t_{f}^{-}$. By simple calculation, we know (16) is held; thus the inputs of all the agents are bounded. In addition, comparing the cases of $t_{f}=10$ and $t_{f}=1$, one can find that the dynamics of the agents' states adapt the preset finite time $t_{f}$ while the inputs of the agents increase linearly with the decreasing of $t_{f}$.

Next, we consider the second-order dynamics case. Control protocol (27) with (39) is applied as $c=2$. We run the simulations with $t_{f}=10$ and $t_{f}=1$, respectively, and show the results in Figure 3. In the simulations, the initial states and velocities are generated randomly. Similarly, we use the same initial states and velocities in both simulations. The simulation results have shown that the groups of agents can reach consensus as $t \rightarrow t_{f}^{-}$. In addition, one can check that the smaller $t_{f}$ will not affect the effectiveness and the performance of our control protocols.

\section{Conclusion}

Finite-time consensus problems for multiagent systems have been investigated in this paper. We have considered both of the first-order and second-order cases. Time-varying linear feedback control protocols have been established under which the systems achieve consensus at any preset time. The condition on the communication topology has been proven to be the same as those in the asymptotical consensus case. The future work includes finite time with switching topology or time-delay. 


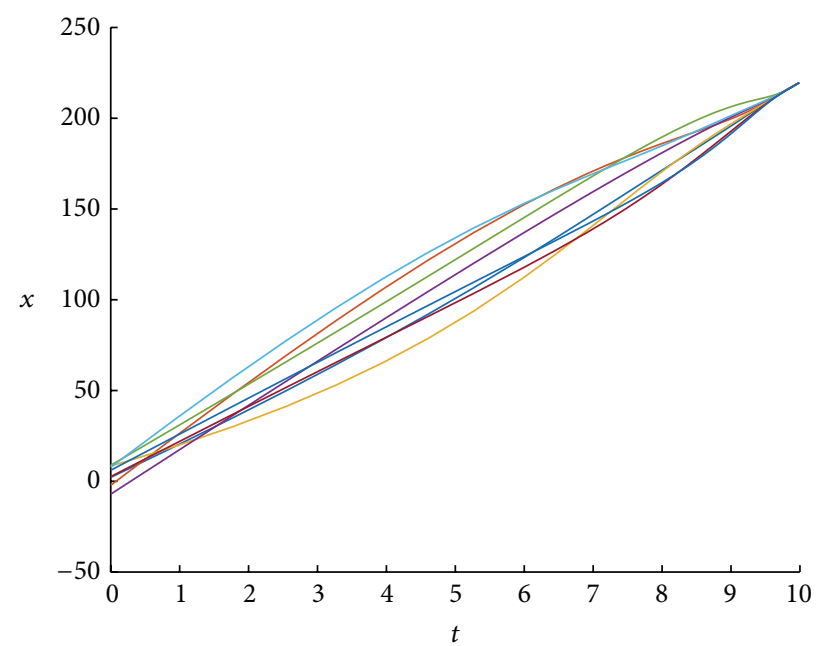

(a) $t_{f}=10$

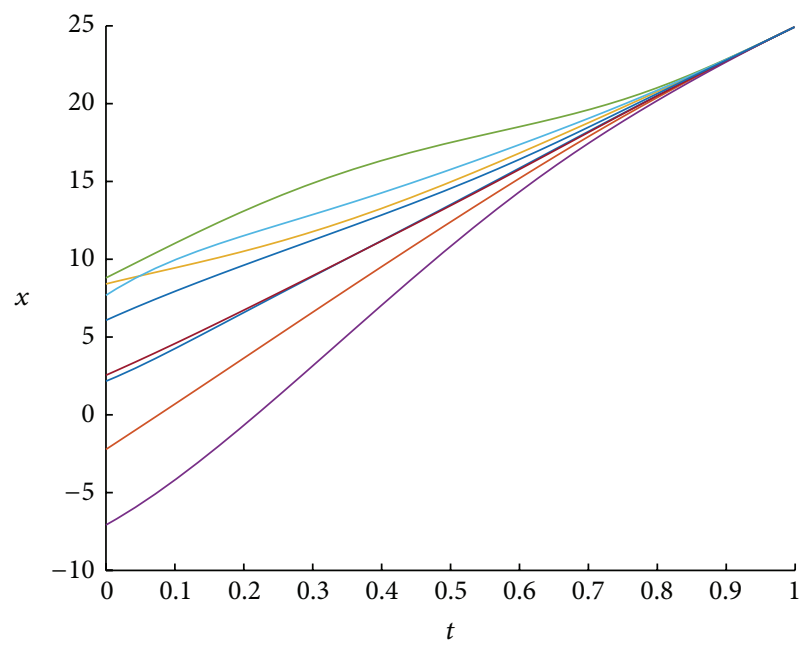

(c) $t_{f}=1$

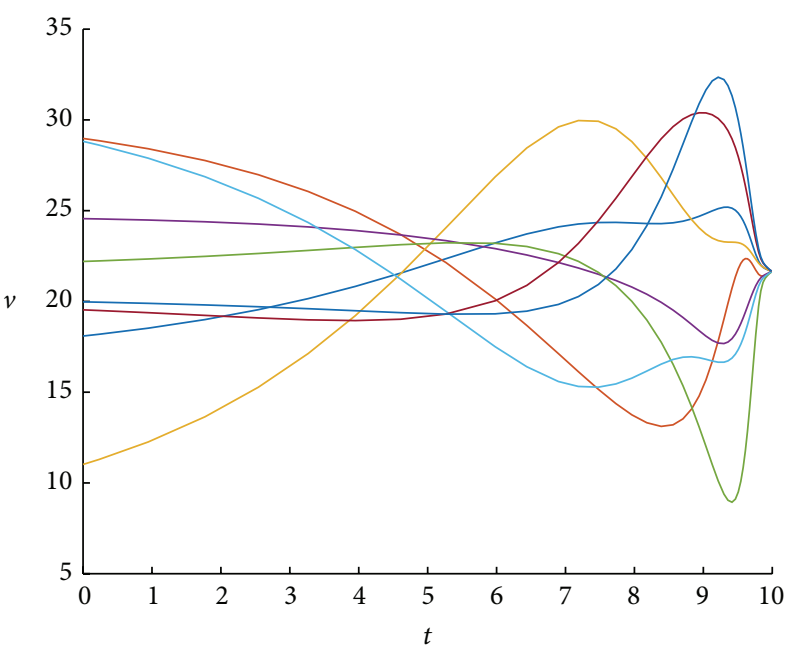

(b) $t_{f}=10$

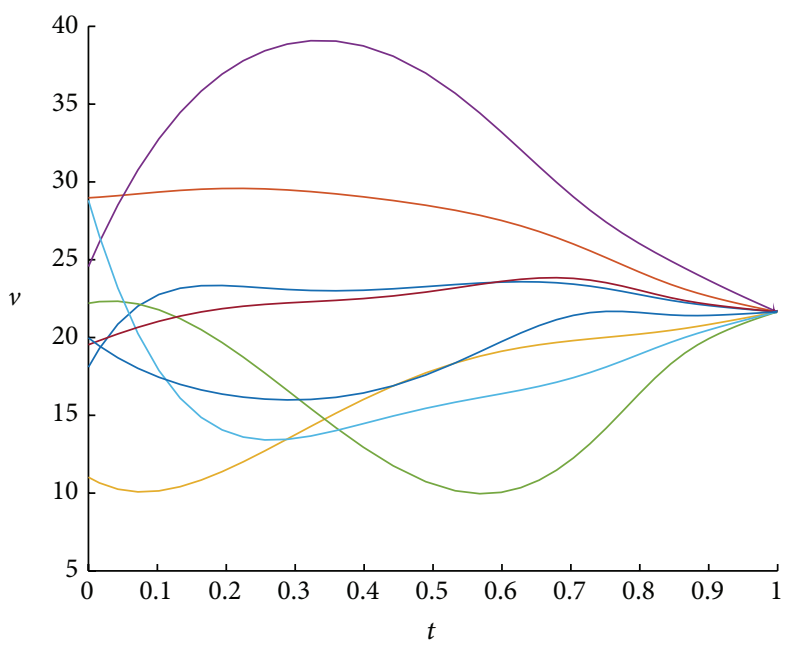

(d) $t_{f}=1$

Figure 3: Simulation results of control protocol (27) with (39) for the double-integrator dynamics case when $c=2$. ((a) and (b)) The case with $t_{f}=10 ;((\mathrm{c})$ and $(\mathrm{d}))$ the case with $t_{f}=1 ;((\mathrm{a})$ and $(\mathrm{c}))$ the states of all the eight agents; ((b) and (d)) the velocities of all the eight agents.

\section{Competing Interests}

The authors declare that there are no competing interests regarding the publication of this paper.

\section{Acknowledgments}

This work was supported in part by grants from the National Natural Science Foundation of China (NSFC, nos. 51575005, 61563006, and 61503008), the China Postdoctoral Science Foundation (no. 2015M570013), the Key Scientific Research Project of Guangxi Education Department (no. ZD2014073), and the Natural Science Foundation of Guangxi Province of China (Grant no. 2014GXNSFBA118284).

\section{References}

[1] W. Ren, R. W. Beard, and E. M. Atkins, "Information consensus in multivehicle cooperative control," IEEE Control Systems Magazine, vol. 27, no. 2, pp. 71-82, 2007.

[2] R. Olfati-Saber, J. A. Fax, and R. M. Murray, "Consensus and cooperation in networked multi-agent systems," Proceedings of the IEEE, vol. 95, no. 1, pp. 215-233, 2007.

[3] T. Vicsek, A. Czirok, E. B. Jacob, I. Cohen, and O. Schochet, "Novel type of phase transition in a system of self-driven particles," Physical Review Letters, vol. 75, no. 6, pp. 1226-1229, 1995.

[4] A. Jadbabaie, J. Lin, and A. S. Morse, "Coordination of groups of mobile autonomous agents using nearest neighbor rules," IEEE Transactions on Automatic Control, vol. 48, no. 6, pp. 988-1001, 2003. 
[5] R. Olfati-Saber and R. M. Murray, "Consensus problems in networks of agents with switching topology and time-delays," IEEE Transactions on Automatic Control, vol. 49, no. 9, pp. 1520$1533,2004$.

[6] W. Ren and R. Beard, "Consensus seeking in multi-agent systems using dynamically changing interaction topologies," IEEE Transactions on Automatic Control, vol. 50, no. 5, pp. 655$661,2005$.

[7] L. Moreau, "Stability of multiagent systems with timedependent communication links," IEEE Transactions on Automatic Control, vol. 50, no. 2, pp. 169-182, 2005.

[8] Z. Lin, B. Francis, and M. Maggiore, "State agreement for continuous-time coupled nonlinear systems," SIAM Journal on Control and Optimization, vol. 46, no. 1, pp. 288-307, 2007.

[9] K. You, Z. Li, and L. Xie, "Consensus condition for linear multiagent systems over randomly switching topologies," Automatica, vol. 49, no. 10, pp. 3125-3132, 2013

[10] Y. Su and J. Huang, "Stability of a class of linear switching systems with applications to two consensus problems," IEEE Transactions on Automatic Control, vol. 57, no. 6, pp. 1420-1430, 2012.

[11] K. Liu, G. Xie, and L. Wang, "Consensus for multi-agent systems under double integrator dynamics with time-varying communication delays," International Journal of Robust and Nonlinear Control, vol. 22, no. 17, pp. 1881-1898, 2012.

[12] K. Liu, G. Xie, and L. Wang, "Containment control for secondorder multi-agent systems with time-varying delays," Systems \& Control Letters, vol. 67, no. 1, pp. 24-31, 2014.

[13] H. Zhang and F. L. Lewis, "Adaptive cooperative tracking control of higher-order nonlinear systems with unknown dynamics," Automatica, vol. 48, no. 7, pp. 1432-1439, 2012.

[14] B. Liu, X. Wang, H. Su, Y. Gao, and L. Wang, "Adaptive secondorder consensus of multi-agent systems with heterogeneous nonlinear dynamics and time-varying delays," Neurocomputing, vol. 118, pp. 289-300, 2013.

[15] H.-X. Hu, A. Liu, Q. Xuan, L. Yu, and G. Xie, "Secondorder consensus of multi-agent systems in the cooperationcompetition network with switching topologies: a time-delayed impulsive control approach," Systems and Control Letters, vol. 62, no. 12, pp. 1125-1135, 2013.

[16] G. Xie and L. Wang, "Consensus control for a class of networks of dynamic agents," International Journal of Robust and Nonlinear Control, vol. 17, no. 10-11, pp. 941-959, 2007.

[17] L. Xiao and S. Boyd, "Fast linear iterations for distributed averaging," Systems \& Control Letters, vol. 53, no. 1, pp. 65-78, 2004.

[18] J. Cortés, "Finite-time convergent gradient flows with applications to network consensus," Automatica, vol. 42, no. 11, pp. 1993-2000, 2006.

[19] S. Y. Khoo, L. X. Xie, and Z. H. Man, "Robust finite-time consensus tracking algorithm for multirobot systems," IEEE/ASME Transactions on Mechatronics, vol. 14, no. 2, pp. 219-228, 2009.

[20] F. Xiao, L. Wang, J. Chen, and Y. Gao, "Finite-time formation control for multi-agent systems," Automatica, vol. 45, no. 11, pp. 2605-2611, 2009.

[21] L. Wang and F. Xiao, "Finite-time consensus problems for networks of dynamic agents," IEEE Transactions on Automatic Control, vol. 55, no. 4, pp. 950-955, 2010.

[22] F. Jiang and L. Wang, "Finite-time weighted average consensus with respect to a monotonic function and its application," Systems \& Control Letters, vol. 60, no. 9, pp. 718-725, 2011.
[23] N. Biggs, Algebraic Graph Theory, Cambridge University Press, Cambridge, UK, 1974.

[24] E. Kreyszig, Advanced Engineering Mathematics, John Wiley \& Sons, New York, NY, USA, 7th edition, 1993. 


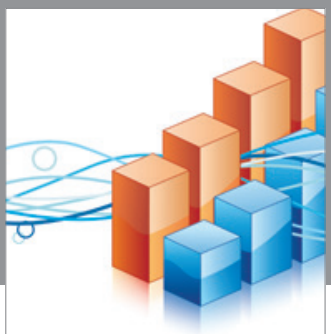

Advances in

Operations Research

vatem alat4

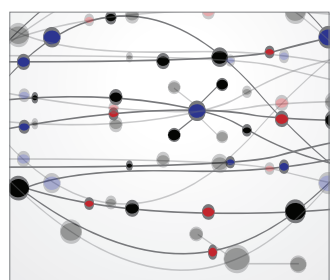

\section{The Scientific} World Journal
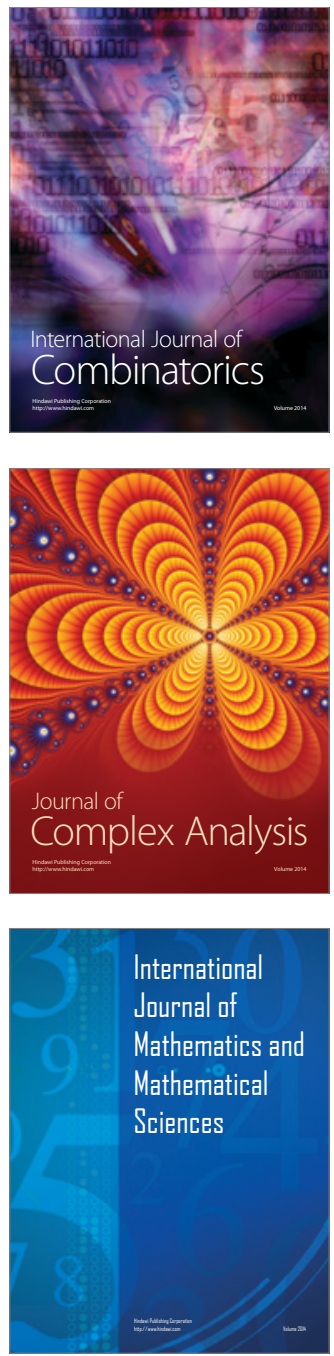
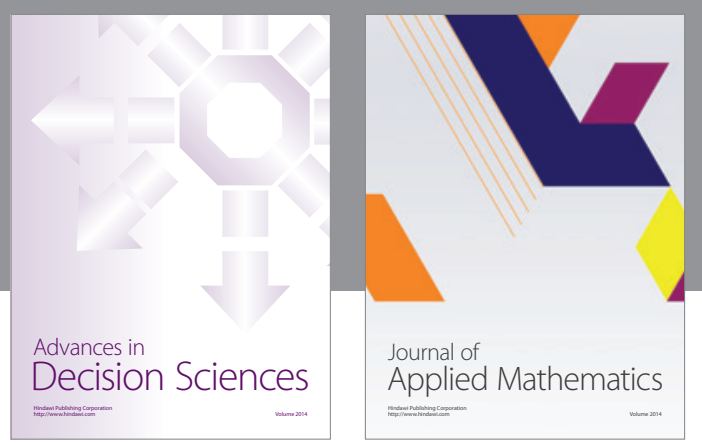

Algebra

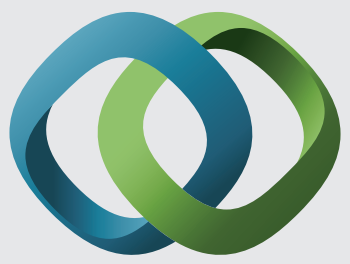

\section{Hindawi}

Submit your manuscripts at

http://www.hindawi.com
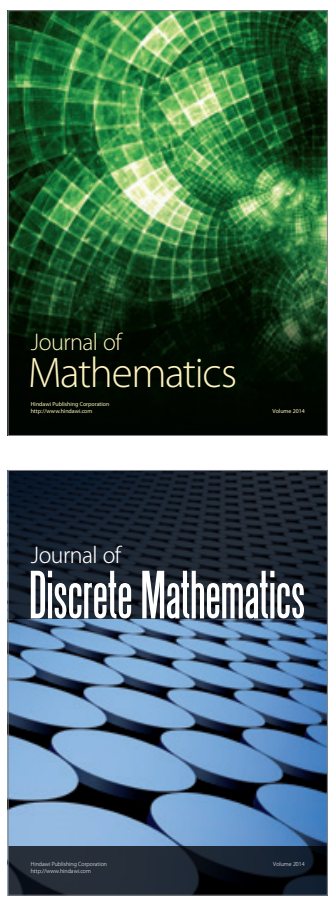

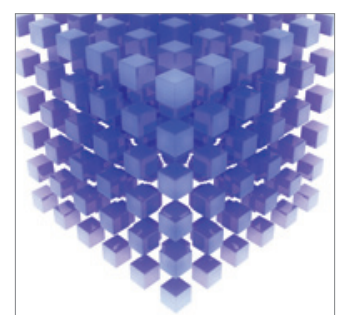

Mathematical Problems in Engineering
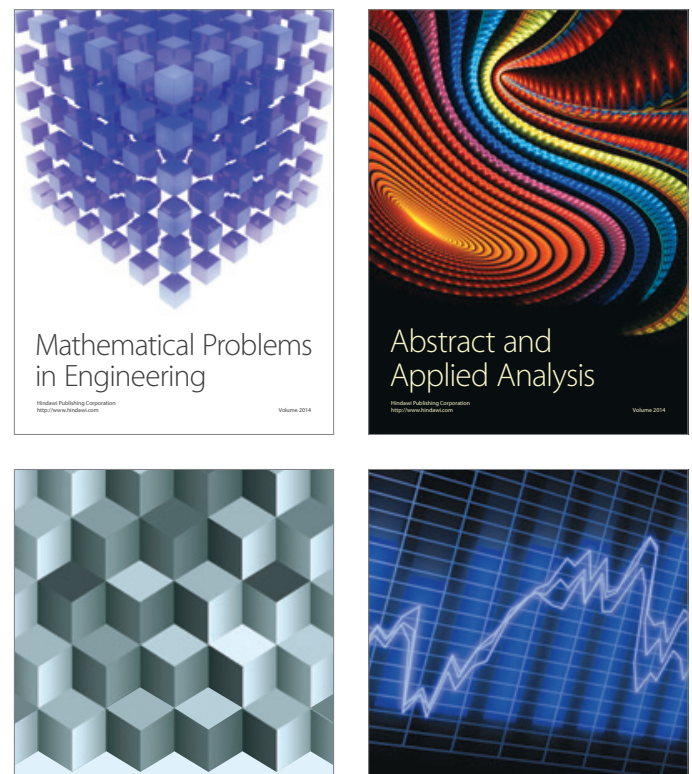

Journal of

Function Spaces

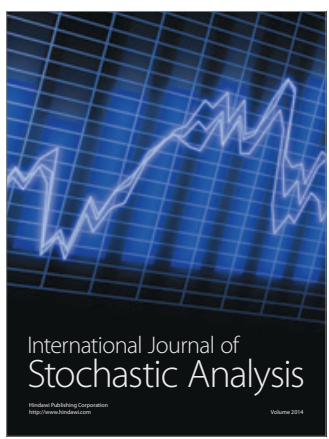

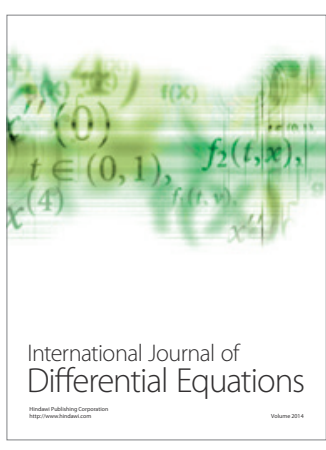
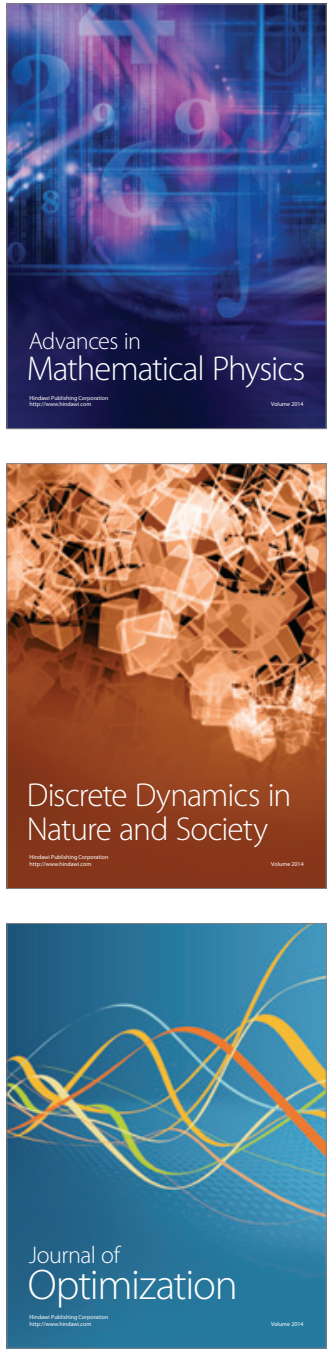